\title{
Spinor Representations of Involute Evolute Curves in $\mathbb{E}^{3}$
}

\author{
Tülay Erişirir $^{1 *}$ and Neslihan Cansu Kardağ ${ }^{1}$ \\ ${ }^{1}$ Department of Mathematics, Faculty of Science and Arts, Erzincan Binali Yildirim University, Erzincan, Turkey \\ ${ }^{*}$ Corresponding author E-mail: tulay.erisir@erzincan.edu.tr
}

Article Info

Keywords: Involute Evolute curves, Spinors

2010 AMS: 15A66, 53A04

Received: 09 May 2019

Accepted: 12 November 2019

Available online: 20 December 2019

\begin{abstract}
In this paper, we have obtained spinor with two complex components representations of Involute Evolute curves in $\mathbb{E}^{3}$. Firstly, we have given the spinor equations of Frenet vectors of two curves which are parameterized by arc-length and have an arbitrary parameter. Moreover, we have chosen that these curves are Involute Evolute curves and have matched these curves with different spinors. Then, we have investigated the answer of question "How are the relationships between the spinors corresponding to the Involute Evolute curves in $\mathbb{E}^{3}$ ?". Finally, we have given an example which crosscheck to theorems throughout this study.
\end{abstract}

\section{Introduction}

The theory of spinors, especially used in applications to electron spin and theory of relativity in quantum mechanics, was expressed by B. L. van der Waerden in 1929. The introduction of spinors is one of the most difficult topics in quantum mechanics. Even if the spin-1/2 is considered, some fundamental sections of spinors, such as the effects of rotation on spinors, turn out to be difficult to explain. Spinors appear to be closely related to the theory of the electromagnetic theory. According to physicists spinors are multilinear transformations. Thanks to this feature, spinors are mathematical entities somewhat like tensors and allow a more general treatment of the notion of invariance under rotation and Lorentz boosts. Spinors can also be used without reference to relativity, but they arise naturally in discussions of the Lorentz group. Moreover, for a spinor it can be say that it is the most basic sort of mathematical object that can be Lorentz-transformed, [1]-[3]. On the other hand, the basic knowledge of spinor theory is based on earlier years, indeed, if we consider the relationship between spinors and Euler's parameters it is date back to 1776. Spinors are vectorial objects and there is no their multilinear features for mathematicians. Also, spinors have one-index. In discussing vectors and tensors there are two ways in which we can proceed; the geometrical and analytical. To use the geometrical approach, we describe each kind of quantity in terms of its magnitudes and directions; in the analytical treatment, we use components.

Spinors were first studied by Elie Cartan in a geometrical sense. Cartan was one of the founders of Lie group theory which is one of the most important topics of mathematics and which has many physical applications. So, Cartan's study is a very impressive reference in terms of the geometry of the spinors since this gives the spinor representation of the basic geometric definitions [1]. In geometrical meaning, another study was made by Vivarelli. In that study, Vivarelli established a one-to-one linear relationship between the quaternions and spinors. In addition, using the relationship between the rotations in quaternions and three-dimensional Euclidean space, Vivarelli actually obtained the spinor representation of the rotations in Euclidean space, [4]. Castillo, on the other hand, examined the spinor formulation of the curve theory, one of the important subjects of differential geometry. In that study, Castillo gave a spinor corresponding to a mutually orthogonal vector triads in three-dimensional Euclidean space and thus obtained a spinor representation of the Frenet frame and curvatures of a curve, [5]. Based on that study, Kişi and Tosun obtained the spinor formulation of the Darboux frame on a directed surface in three-dimensional Euclidean space, [6]. Similarly, in [7], the spinor Bishop equations of the curves in $\mathbb{E}^{3}$ have been expressed.

Ketenci et. al investigated the answer of question "How does a spinor correspond to a mutually orthogonal vector triad in three dimensional Minkowski space $\mathbb{E}_{1}^{3}$ ?". Thus, they introduced hyperbolic spinors. Based on this, they matched the hyperbolic spinors which have hyperbolic components up with Frenet frame of a curve in Minkowski space $\mathbb{E}_{1}^{3}$, [8]. Then, Erişir et. al obtained the spinor representation of the Bishop frame, an alternative frame, of a curve in the three-dimensional Minkowski space, and the spinor formulation of the relationship between

Email addresses and ORCID numbers: tulay.erisir@erzincan.edu.tr, https://orcid.org/0000-0001-6444-1460 (T. Erişir), Neslihan.2424@outlook.com, https://orcid.org/0000-0002-8505-3819 (N. C. Kardağ) 
Frenet frame and Bishop frame, [9]. Also, the Darboux frame on the oriented surface in $\mathbb{E}_{1}^{3}$ was obtained by the aid of hyperbolic spinors, [10]. Moreover, in [11] Tarakçığlu et. al considered the Vivarelli's study and they gave a different approach to the relationship between the split quaternions and rotations in Minkowski space $\mathbb{R}_{1}^{3}$. In addition, they obtained an automorphism of the split quaternion algebra $\mathrm{H}^{\prime}$ corresponding to a rotation in $\mathbb{R}_{1}^{3}$. Then, they gave the relationship between the hyperbolic spinors and rotations in $\mathbb{R}_{1}^{3}$.

In this paper, we have studied on spinors with two complex components and we have given spinor representations of Involute Evolute curves in $\mathbb{E}^{3}$. Firstly, we have introduced spinor representation of Frenet vectors of any unit-speed curve in three dimensional Euclidean space $\mathbb{E}^{3}$. Then, we have obtained spinor equations of the curve which is not parameterized by arc-length and considered the Involute Evolute curves corresponding the different spinors. Thus, we have investigated the answer of question "How are the relations between the spinors corresponding to the Involute Evolute curves in $\mathbb{E}^{3}$ ?". Finally, we have given an example.

\section{Preliminaries}

\subsection{Involute Evolute curves in $\mathbb{E}^{3}$}

It is well known that if a curve is differentiable at the each point of an open interval then a set of mutually orthogonal unit vectors can be constructed. These vectors are called tangent $\boldsymbol{T}$, normal $\boldsymbol{N}$ and binormal $\boldsymbol{B}$ unit vectors or the Serret-Frenet frame, collectively. So, let us consider that the regular curve $(\alpha)$ which is the differentiable function so that $\alpha: I \rightarrow \mathbb{E}^{3},(I \subseteq \mathbb{R})$ has the arbitrary parameter $t$. Moreover, for $\forall t \in I$ the Frenet vectors on the point $\alpha(t)$ of the curve $(\alpha)$ are given by $\{\boldsymbol{T}(t), \boldsymbol{N}(t), \boldsymbol{B}(t)\}$. So, these Frenet vectors are obtained by the following equations

$$
\begin{aligned}
& \boldsymbol{T}=\frac{1}{\left\|\alpha^{\prime}\right\|} \alpha^{\prime}, \\
& \boldsymbol{N}=\boldsymbol{B} \times \boldsymbol{T}, \\
& \boldsymbol{B}=\frac{1}{\left\|\alpha^{\prime} \times \alpha^{\prime \prime}\right\|}\left(\alpha^{\prime} \times \alpha^{\prime \prime}\right)
\end{aligned}
$$

where "' " is the derivative with respect to arbitrary parameter $t, \kappa$ and $\tau$ are the curvature and torsion of this curve $(\alpha),[12]$.

Moreover, the Frenet derivative formulas of this curve $(\alpha)$ are given by

$$
\begin{aligned}
& \boldsymbol{T}^{\prime}=\left\|\alpha^{\prime}\right\| \kappa \boldsymbol{N}, \\
& \boldsymbol{N}^{\prime}=\left\|\alpha^{\prime}\right\|(-\kappa \boldsymbol{T}+\tau \boldsymbol{B}), \\
& \boldsymbol{B}^{\prime}=-\left\|\alpha^{\prime}\right\| \tau \boldsymbol{N}
\end{aligned}
$$

[12].

Now, we know that if there is equation $\left\|\alpha^{\prime}(s)\right\|=1$ for $\forall s \in I$ on the point $\alpha(s)$ of the curve $\alpha: I \rightarrow \mathbb{E}^{3},(I \subseteq \mathbb{R})$, the curve $(\alpha)$ is called as the curve parameterized by arc-length parameter $s$. So, the Frenet vectors of the curve $(\alpha)$ parameterized by arc-length parameter can be obtained by

$$
\begin{aligned}
& \boldsymbol{T}=\alpha^{\prime}, \\
& \boldsymbol{N}=\frac{1}{\left\|\alpha^{\prime \prime}\right\|} \alpha^{\prime \prime}, \\
& \boldsymbol{B}=\boldsymbol{T} \times \boldsymbol{N}
\end{aligned}
$$

where " $"$ is the derivative with respect to arc-length parameter $s$. Moreover, the Frenet formulas of this curve are as

$$
\begin{aligned}
& \boldsymbol{T}^{\prime}=\kappa \boldsymbol{N}, \\
& \boldsymbol{N}^{\prime}=-\kappa \boldsymbol{T}+\tau \boldsymbol{B}, \\
& \boldsymbol{B}^{\prime}=-\tau \boldsymbol{N},
\end{aligned}
$$

[12].

The Involute Evolute curves in $\mathbb{E}^{3}$ are well known and one of the most studied curve pairs in elementary differential geometry. So, for the Involute Evolute curves, the following definition and theorems can be given.

Definition 2.1. Let the curve $\alpha: I \rightarrow \mathbb{E}^{3}$ be parameterized by arc-length parameter and the curve $\beta: I \rightarrow \mathbb{E}^{3}$ be any curve which has an arbitrary parameter. Moreover, the Frenet frames of these curves $(\alpha)$ and $(\beta)$ are considered that $\{\boldsymbol{T}, \boldsymbol{N}, \boldsymbol{B}\}$ and $\left\{\boldsymbol{T}^{*}, \boldsymbol{N}^{*}, \boldsymbol{B}^{*}\right\}$, respectively. So, if there is equation

$$
\left\langle\boldsymbol{T}, \boldsymbol{T}^{*}\right\rangle=0,
$$

then the curve $(\beta)$ is called the involute of the curve $(\alpha)$ and the curve $(\alpha)$ is called the evolute of the curve $(\beta),[12]$.

Theorem 2.2. Let the curve $\beta, \alpha: I \rightarrow \mathbb{E}^{3}$ be consider Involute Evolute curves, respectively. Then, the distance between mutual points of these curves is

$$
d(\alpha(s), \beta(s))=|c-s|
$$

So, it can be written

$$
\beta(s)=\alpha(s)+(c-s) \boldsymbol{T}(s)
$$


Theorem 2.3. Let the Frenet frames of the Involute Evolute curves $(\beta, \alpha)$ be $\left\{\boldsymbol{T}^{*}, \boldsymbol{N}^{*}, \boldsymbol{B}^{*}\right\}$ and $\{\boldsymbol{T}, \boldsymbol{N}, \boldsymbol{B}\}$, respectively. So, the relationship between these Frenet frames is

$$
\begin{aligned}
& \boldsymbol{T}^{*}=\boldsymbol{N}, \\
& \boldsymbol{N}^{*}=\frac{1}{\sqrt{\kappa^{2}+\tau^{2}}}(-\kappa \boldsymbol{T}+\tau \boldsymbol{B},) \\
& \boldsymbol{B}^{*}=\frac{1}{\sqrt{\kappa^{2}+\tau^{2}}}(\tau \boldsymbol{T}+\kappa \boldsymbol{B},)
\end{aligned}
$$

[12].

Theorem 2.4. Let the Frenet curvatures of the Involute Evolute curves $(\beta, \alpha)$ be $\kappa^{*}, \tau^{*}$ and $\kappa, \tau$, respectively. So, the relationship between these Frenet curvatures is

$$
\begin{aligned}
\kappa^{*} & =\frac{\sqrt{\kappa^{2}+\tau^{2}}}{|(c-s) \kappa|} \\
\tau^{*} & =\frac{\kappa \tau^{\prime}-\kappa^{\prime} \tau}{|(c-s) \kappa|\left(\kappa^{2}+\tau^{2}\right)}
\end{aligned}
$$

[12].

\subsection{Spinors}

In this section, spinors introduced by Cartan [1], which is a basic study in geometric sense, are given. Afterwards, the spinors in the study given by Del Castillo and Barrales are mentioned, [5].

Consider that $\boldsymbol{x}=\left(x_{1}, x_{2}, x_{3}\right) \in \mathbb{C}^{3}$ is the isotropic vector and $\mathbb{C}^{3}$ is the three-dimensional complex vector space. So, we obtain $x_{1}^{2}+x_{2}^{2}+x_{3}^{2}=0$. The set of isotropic vectors in the vector space $\mathbb{C}^{3}$ forms a two-dimensional surface in the space $\mathbb{C}^{2}$. If this two-dimensional surface is parameterized by $\xi_{1}$ and $\xi_{2}$ coordinates, then $x_{1}=\xi_{1}^{2}-\xi_{2}^{2}, x_{2}=i\left(\xi_{1}^{2}+\xi_{2}^{2}\right), x_{3}=-2 \xi_{1} \xi_{2}$ is obtained. It is seen from the solution of this equation that $\xi_{1}= \pm \sqrt{\frac{x_{1}-i x_{2}}{2}}, \xi_{2}= \pm \sqrt{\frac{-x_{1}-i x_{2}}{2}}$. It is seen that; in the complex vector space $\mathbb{C}^{3}$, each isotropic vector corresponds to two vectors, $\left(\xi_{1}, \xi_{2}\right)$ and $\left(-\xi_{1},-\xi_{2}\right)$ in the space $\mathbb{C}^{2}$. Conversely; both vectors so given in space $\mathbb{C}^{2}$ correspond to the same isotropic vector $\boldsymbol{x}$. Cartan expressed that the two-dimensional complex vectors $\xi=\left(\xi_{1}, \xi_{2}\right)$ described in this way are called as spinors. In addition, Cartan emphasized that spinors are not only two-dimensional complex vectors, but also represent a three-dimensional complex isotropic vectors, [1].

Consider the $S O(3)$, the group of rotations around the origin in the three-dimensional real vector space $\mathbb{R}^{3}$, and the $S U(2)$, the group of $2 \times 2$ dimensional unitary matrices. As is known, the $S O(3)$ group is homomorphic to the $S U(2)$ group, [5, 13, 14]. By means of this homomorphism and spinors introduced by Cartan, the isotropic vector $\boldsymbol{a}+i \boldsymbol{b}$ is matched with spinor

$$
\xi=\left(\begin{array}{l}
\xi_{1} \\
\xi_{2}
\end{array}\right)
$$

where $\boldsymbol{a}, \boldsymbol{b} \in \mathbb{R}^{3}$. So, we have $x_{1}=\xi_{1}^{2}-\xi_{2}^{2}, x_{2}=i\left(\xi_{1}^{2}+\xi_{2}^{2}\right)$ and $x_{3}=-2 \xi_{1} \xi_{2}$ where $\boldsymbol{a}+i \boldsymbol{b}=\left(x_{1}, x_{2}, x_{3}\right) \in \mathbb{C}^{3}$, [1, 5]. As it is known, Pauli matrices form a basis for $2 x 2$-dimensional Hermitian and unitary matrices. Using the Pauli matrices and the matrix $C=\left(\begin{array}{cc}0 & 1 \\ -1 & 0\end{array}\right)$, the matrices $\sigma$ are generated as $\sigma_{1}=\left(\begin{array}{cc}1 & 0 \\ 0 & -1\end{array}\right), \sigma_{2}=\left(\begin{array}{cc}i & 0 \\ 0 & i\end{array}\right)$ and $\sigma_{3}=\left(\begin{array}{cc}0 & -1 \\ -1 & 0\end{array}\right),[5,15]$. On the other hand, the mate $\hat{\xi}$ of the spinor $\xi$ is obtained as

$$
\hat{\xi}=-\left(\begin{array}{cc}
0 & 1 \\
-1 & 0
\end{array}\right) \bar{\xi}=-\left(\begin{array}{cc}
0 & 1 \\
-1 & 0
\end{array}\right)\left(\begin{array}{l}
\overline{\xi_{1}} \\
\xi_{2}
\end{array}\right)=\left(\begin{array}{c}
-\overline{\xi_{2}} \\
\overline{\xi_{1}}
\end{array}\right)
$$

Throughout this information, we obtain that

$$
\begin{aligned}
& \boldsymbol{a}+i \boldsymbol{b}=\xi^{t} \sigma \xi \\
& \boldsymbol{c}=-\hat{\xi}^{t} \sigma \xi
\end{aligned}
$$

where $\boldsymbol{a}+\boldsymbol{i} \boldsymbol{b}$ is the isotropic vector in the space $\mathbb{C}^{3}$ and $\boldsymbol{c} \in \mathbb{R}^{3},[5]$.

When necessary operations are considered, it is seen that the vectors $\boldsymbol{a}, \boldsymbol{b}$ and $\boldsymbol{c}$ are equal in length and these vectors are mutually orthogonal, [5]. Moreover, the following proposition can be given.

Proposition 2.5. Let two arbitrary spinors be $\xi$ and $\phi$. Then, the following statements are hold;

$$
\begin{array}{ll}
\text { i) } & \overline{\phi^{t} \sigma \xi}=-\hat{\phi}^{t} \sigma \hat{\xi} \\
\text { ii) } & \lambda \hat{\phi+\mu} \xi=\bar{\lambda} \hat{\phi}+\bar{\mu} \hat{\xi} \\
\text { iii) } & \hat{\boldsymbol{\xi}}=-\xi \\
\text { iv) } & \phi^{t} \sigma \xi=\xi \xi^{t} \sigma \phi
\end{array}
$$


Now, let a curve parameterized by arc-length be $\alpha: I \rightarrow \mathbb{E}^{3},(I \subseteq \mathbb{R})$. So, $\left\|\alpha^{\prime}(s)\right\|=1$ where $s$ is the arc-length parameter of the curve $(\alpha)$. Moreover, we consider that the Frenet vectors of this curve are $\{\boldsymbol{N}, \boldsymbol{B}, \boldsymbol{T}\}$ and the spinor $\xi$ corresponds to the Frenet vectors $\{\boldsymbol{N}, \boldsymbol{B}, \boldsymbol{T}\}$. Thus, the following equations

$$
\begin{aligned}
\boldsymbol{N}+i \boldsymbol{B} & =\xi^{t} \sigma \xi=\left(\xi_{1}{ }^{2}-\xi_{2}^{2}, i\left(\xi_{1}^{2}+\xi_{2}^{2}\right),-2 \xi_{1} \xi_{2}\right) \\
\boldsymbol{T} & =-\widehat{\xi}^{t} \sigma \xi=\left(\xi_{1} \bar{\xi}_{2}+\overline{\xi_{1}} \xi_{2}, i\left(\xi_{1} \overline{\xi_{2}}-\overline{\xi_{1}} \xi_{2}\right),\left|\xi_{1}\right|^{2}-\left|\xi_{2}\right|^{2}\right)
\end{aligned}
$$

can be written where $\bar{\xi}^{t} \xi=1$ since these vectors are mutually orthogonal, [5]. Moreover, the following theorem can be given.

Theorem 2.6. If the spinor $\xi$ with two complex components represents the triad $\{\boldsymbol{N}, \boldsymbol{B}, \boldsymbol{T}\}$ of a curve parameterized by its arc-length s the Frenet equations are equivalent to the single spinor equation

$$
\frac{d \xi}{d s}=\frac{1}{2}(-i \tau \xi+\kappa \hat{\xi})
$$

where $\kappa$ and $\tau$ denote the curvature and torsion of the curve, respectively, [5].

\section{Main theorems and proofs}

In this section, first of all, we have expressed that the spinor representations of each Frenet vectors $\{\boldsymbol{N}, \boldsymbol{B}, \boldsymbol{T}\}$ of a unit-speed curve $(\alpha)$, separately. In addition that, we have considered that the curve $(\beta)$ which has not arc-length parameter and a different spinor is corresponded to the Frenet vectors $\left\{\boldsymbol{N}^{*}, \boldsymbol{B}^{*}, \boldsymbol{T}^{*}\right\}$ of this curve. Moreover, we have given the spinor equations of this curve. Then, we have regarded that the curves $(\beta, \alpha)$ are Involute Evolute curves and obtained the relationship between spinors corresponding to these curves with theorems. Finally, we have given an example.

Let $\alpha: \mathrm{I} \rightarrow \mathbb{E}^{3}$ be arbitrary unit-speed curve and the Frenet vectors of this curve be $\{\boldsymbol{N}, \boldsymbol{B}, \boldsymbol{T}\}$. So, the following theorem can be given.

Theorem 3.1. Let the Frenet vectors of the unit-speed curve $\alpha: I \rightarrow \mathbb{E}^{3}$ be $\{\boldsymbol{N}, \boldsymbol{B}, \boldsymbol{T}\}$. We assume that the spinor $\xi$ is corresponded to this curve $(\alpha)$, So, the spinor equations of these Frenet vectors are

$$
\begin{aligned}
& \boldsymbol{T}=-\hat{\xi}^{t} \sigma \xi \\
& \boldsymbol{N}=\frac{1}{2}\left(\xi^{t} \sigma \xi-\hat{\xi}^{t} \sigma \hat{\xi}\right), \\
& \boldsymbol{B}=-\frac{i}{2}\left(\xi^{t} \sigma \xi+\hat{\xi}^{t} \sigma \hat{\xi}\right) .
\end{aligned}
$$

Proof. Let the spinor $\xi$ be correspond to the Frenet curve $\{\boldsymbol{N}, \boldsymbol{B}, \boldsymbol{T}\}$ of unit-speed curve $(\alpha)$. Then, considering the equations (2.2) and (2.6) for the tangent vector on the point $\alpha(s)$ of $(\alpha)$ the following equation

$$
\boldsymbol{T}=\alpha^{\prime}=-\hat{\xi}^{t} \sigma \xi
$$

can be written. If we calculate the derivative of the equation (3.1) and make necessary arrangement, we obtain that

$$
\alpha^{\prime \prime}=\frac{\kappa}{2}\left(\xi^{t} \sigma \xi-\hat{\xi}^{t} \sigma \hat{\xi}\right)
$$

On the other hand, let us consider the equation (2.2), So, we obtain that the spinor equation of the normal vector $N$ of the curve $(\alpha)$ is obtain that

$$
N=\frac{1}{2}\left(\xi^{t} \sigma \xi-\hat{\xi}^{t} \sigma \hat{\xi}\right)
$$

Similarly, using the equations (2.3), (3.1) and (3.2), we have

$$
N^{\prime}=\frac{1}{2}\left[-i \tau\left(\xi^{t} \sigma \xi+\hat{\xi}^{t} \sigma \hat{\xi}\right)+2 \kappa \hat{\xi}^{t} \sigma \xi\right]
$$

and

$$
\frac{1}{2}\left[-i \tau\left(\xi^{t} \sigma \xi+\hat{\xi}^{t} \sigma \hat{\xi}\right)+2 \kappa \hat{\xi}^{t} \sigma \xi\right]=-\kappa\left(-\hat{\xi}^{t} \sigma \xi\right)+\tau \boldsymbol{B} .
$$

And finally, the spinor equation of the binormal tangent of the curve $(\alpha)$ is

$$
\boldsymbol{B}=-\frac{i}{2}\left(\xi^{t} \sigma \xi+\hat{\xi}^{t} \sigma \hat{\xi}\right)
$$

So, the proof ends.

Indeed, if we consider the first equality in the equation (2.6), we see that $N=\operatorname{Re}\left(\xi^{t} \sigma \xi\right)$ and $\boldsymbol{B}=\operatorname{Im}\left(\xi^{t} \sigma \xi\right)$. So, considering complex numbers we obtain that

$$
\begin{aligned}
& \boldsymbol{N}=\frac{1}{2}\left(\xi^{t} \sigma \xi+\overline{\xi^{t} \sigma \xi}\right), \\
& \boldsymbol{B}=-\frac{i}{2}\left(\xi^{t} \sigma \xi-\overline{\xi^{t} \sigma \xi}\right) .
\end{aligned}
$$


Finally, using the Proposition 2.5, we reach the equations (3.2) and (3.3).

Moreover, the spinor equations of these vectors can be written in terms of components as follows since they will be used operations after that

$$
\begin{aligned}
& \boldsymbol{T}=\left(\xi_{1} \overline{\xi_{2}}+\overline{\xi_{1}} \xi_{2}, i\left(\xi_{1} \overline{\xi_{2}}-\overline{\xi_{1}} \xi_{2}\right),\left|\xi_{1}\right|^{2}-\left|\xi_{2}\right|^{2}\right), \\
& \boldsymbol{N}=\frac{1}{2}\left(\xi_{1}^{2}-\xi_{2}^{2}-{\overline{\xi_{2}}}^{2}+\bar{\xi}_{1}^{2}, i\left(\xi_{1}^{2}+\xi_{2}^{2}-{\overline{\xi_{1}}}^{2}-{\overline{\xi_{2}}}^{2}\right),-2 \xi_{1} \xi_{2}-2 \overline{\xi_{1} \xi_{2}}\right), \\
& \boldsymbol{B}=-\frac{i}{2}\left(\xi_{1}{ }^{2}-\xi_{2}{ }^{2}+\bar{\xi}_{2}^{2}-\bar{\xi}_{1}^{2}, i\left(\xi_{1}{ }^{2}+\xi_{2}{ }^{2}+\bar{\xi}_{1}^{2}+\bar{\xi}_{2}^{2}\right),-2 \xi_{1} \xi_{2}+2 \bar{\xi}_{1} \xi_{2}\right) .
\end{aligned}
$$

On the other hand, let us take any curve $(\beta)$ which is not parameterized by arc length. Moreover, let the Frenet vectors of this curve be $\left\{\boldsymbol{N}^{*}, \boldsymbol{B}^{*}, \boldsymbol{T}^{*}\right\}$ and the different spinor corresponding the curve $(\beta)$ be $\phi$. So, similar to the equation (2.6), we can write

$$
\begin{array}{r}
\boldsymbol{N}^{*}+i \boldsymbol{B}^{*}=\phi^{t} \sigma \phi, \\
\boldsymbol{T}^{*}=-\widehat{\phi}^{t} \sigma \phi .
\end{array}
$$

So, the spinor equations of this curve $(\beta)$ can be written by components as

$$
\begin{aligned}
& \boldsymbol{T}^{*}=\left(\phi_{1} \overline{\phi_{2}}+\overline{\phi_{1}} \phi_{2}, i\left(\phi_{1} \overline{\phi_{2}}-\overline{\phi_{1}} \phi_{2}\right),\left|\phi_{1}\right|^{2}-\left|\phi_{2}\right|^{2}\right), \\
& \boldsymbol{N}^{*}=\frac{1}{2}\left({\phi_{1}}^{2}-\phi_{2}{ }^{2}+{\overline{\phi_{1}}}^{2}-{\overline{\phi_{2}}}^{2}, i\left(\phi_{1}^{2}+\phi_{2}{ }^{2}-{\overline{\phi_{1}}}^{2}-{\overline{\phi_{2}}}^{2}\right),-2\left(\phi_{1} \phi_{2}+\overline{\phi_{1} \phi_{2}}\right)\right), \\
& \boldsymbol{B}^{*}=-\frac{i}{2}\left({\phi_{1}}^{2}-{\phi_{2}}^{2}+{\overline{\phi_{2}}}^{2}-{\overline{\phi_{1}}}^{2}, i\left({\phi_{1}}^{2}+\phi_{2}{ }^{2}+{\overline{\phi_{1}}}^{2}+{\overline{\phi_{2}}}^{2}\right),-2\left(\phi_{1} \phi_{2}-\overline{\phi_{1} \phi_{2}}\right)\right)
\end{aligned}
$$

for the curve $(\beta)$. In addition that, the equation (3.5) provides the equation (2.1). So, we can give the following theorem.

Theorem 3.2. Let the Frenet vectors of the curve $(\beta)$ which is not parameterized by arc length be $\left\{\boldsymbol{N}^{*}, \boldsymbol{B}^{*}, \boldsymbol{T}^{*}\right\}$ and the spinor corresponding to this curve be $\phi$. So, the Frenet equation of this curve in terms of a single spinor equation is written by

$$
\frac{d \phi}{d s}=\frac{\left\|\beta^{\prime}\right\|}{2}\left(-i \tau^{*} \phi+\kappa^{*} \hat{\phi}\right)
$$

Proof. Let the Frenet vectors $\left\{\boldsymbol{N}^{*}, \boldsymbol{B}^{*}, \boldsymbol{T}^{*}\right\}$ of the curve $(\beta)$ be correspond to the spinor $\phi$. We know that $\{\phi, \hat{\phi}\}$ is the basis for the spinor with two complex components. So, it can be written

$$
\frac{d \phi}{d s}=f \phi+g \hat{\phi}
$$

where the functions $f$ and $g$ are arbitrary, complex-valued functions. On the other hand, using the equations (2.1), (3.5) and (3.6) we obtain that

$$
\left\|\beta^{\prime}\right\|\left(-\boldsymbol{\kappa}^{*} \boldsymbol{T}^{*}-i \tau^{*}\left(\boldsymbol{N}^{*}+i \boldsymbol{B}^{*}\right)\right)=2 f\left(\boldsymbol{N}^{*}+i \boldsymbol{B}^{*}\right)-2 g \boldsymbol{T}^{*}
$$

So, we have

$$
f=\frac{-i \tau^{*}\left\|\beta^{\prime}\right\|}{2}, \quad g=\frac{\kappa^{*}\left\|\beta^{\prime}\right\|}{2} .
$$

Finally, if we consider the equations (3.6) and (3.7), we obtain that the Frenet vectors of the curve $(\beta)$ in terms of a single spinor equation as

$$
\frac{d \phi}{d s}=\frac{\left\|\beta^{\prime}\right\|}{2}\left(-i \tau^{*} \phi+\kappa^{*} \hat{\phi}\right)
$$

where $\kappa^{*}$ and $\tau^{*}$ are curvature and torsion of curve $(\beta)$.

Now, we express the spinor representation of Involute Evolute curves. Let us consider the curves $\alpha, \beta: I \rightarrow \mathbb{E}^{3}$ and the Frenet vectors $\{\boldsymbol{T}, \boldsymbol{N}, \boldsymbol{B}\}$ and $\left\{\boldsymbol{T}^{*}, \boldsymbol{N}^{*}, \boldsymbol{B}^{*}\right\}$ of the curves $(\alpha)$ and $(\beta)$, respectively. Moreover, the curves $(\beta, \alpha)$ are considered that Involute Evolute curves and the spinors $\phi$ and $\xi$ are corresponded to the Involute Evolute curves $(\beta, \alpha)$, respectively. So, we can give the following theorem.

Theorem 3.3. Let the curves $\beta, \alpha: I \rightarrow \mathbb{E}^{3}$ be Involute Evolute curves which have the Frenet vectors $\left\{\boldsymbol{N}^{*}, \boldsymbol{B}^{*}, \boldsymbol{T}^{*}\right\}$ and $\{\boldsymbol{N}, \boldsymbol{B}, \boldsymbol{T}\}$, respectively. Moreover, the spinors corresponding to the Frenet vectors of these curves $(\alpha)$ and $(\beta)$ are considered as $\xi$ and $\phi$, respectively. So, the relationship between the spinor equations of Involute Evolute curves is

$$
\bar{\phi}^{t} \phi=C \phi \bar{\phi}^{t} \hat{\xi}
$$

where $C=\left(\begin{array}{cc}0 & 1 \\ -1 & 0\end{array}\right)$

Proof. We consider that the curves $\alpha, \beta: I \rightarrow \mathbb{E}^{3}$ are Involute Evolute curves which have the Frenet vectors $\{\boldsymbol{N}, \boldsymbol{B}, \boldsymbol{T}\}$ and $\left\{\boldsymbol{N}^{*}, \boldsymbol{B}^{*}, \boldsymbol{T}^{*}\right\}$, respectively. So, we know that relationship between the tangent vectors of these curves is $\left\langle\boldsymbol{T}, \boldsymbol{T}^{*}\right\rangle=0$. Thus, using this relation and the first equations in the equations (3.4), (3.5), we obtain

$$
\left(\xi_{1} \overline{\xi_{2}}+\overline{\xi_{1}} \xi_{2}\right)\left(\phi_{1} \overline{\phi_{2}}+\overline{\phi_{1}} \phi_{2}\right)-\left(\xi_{1} \overline{\xi_{2}}-\overline{\xi_{1}} \xi_{2}\right)\left(\phi_{1} \overline{\phi_{2}}-\overline{\phi_{1}} \phi_{2}\right)+\left(\left|\xi_{1}\right|^{2}-\left|\xi_{2}\right|^{2}\right)\left(\left|\phi_{1}\right|^{2}-\left|\phi_{2}\right|^{2}\right)=0
$$


and

$$
2 \xi_{1} \overline{\xi_{2}} \overline{\phi_{1}} \phi_{2}+2 \overline{\xi_{1}} \xi_{2} \phi_{1} \overline{\phi_{2}}+\left|\xi_{1}\right|^{2}\left|\phi_{1}\right|^{2}-\left|\xi_{1}\right|^{2}\left|\phi_{2}\right|^{2}-\left|\xi_{2}\right|^{2}\left|\phi_{1}\right|^{2}+\left|\xi_{2}\right|^{2}\left|\phi_{2}\right|^{2}=0 .
$$

Also, if the equation (3.8) is written as matrix product, the following equation can be written

$$
\left(\begin{array}{ll}
\xi_{1} & \xi_{2}
\end{array}\right)\left(\begin{array}{c}
\overline{\phi_{1}} \\
\overline{\phi_{2}}
\end{array}\right)\left(\begin{array}{ll}
\overline{\xi_{1}} & \overline{\xi_{2}}
\end{array}\right)\left(\begin{array}{l}
\phi_{1} \\
\phi_{2}
\end{array}\right)+\left(\begin{array}{ll}
\xi_{1} & \xi_{2}
\end{array}\right)\left(\begin{array}{c}
\phi_{2} \\
-\phi_{1}
\end{array}\right)\left(\begin{array}{cc}
\overline{\phi_{1}} & \overline{\phi_{2}}
\end{array}\right)\left(\begin{array}{c}
\overline{\xi_{2}} \\
-\overline{\xi_{1}}
\end{array}\right)=0
$$

where the spinors $\xi$ and $\phi$ are written as column matrix like these $\xi=\left(\begin{array}{l}\xi_{1} \\ \xi_{2}\end{array}\right)$ and $\phi=\left(\begin{array}{c}\phi_{1} \\ \phi_{2}\end{array}\right)$. Finally, we have

$$
\bar{\phi}^{t} \phi=C \phi \bar{\phi}^{t} \hat{\xi}
$$

where $C=\left(\begin{array}{cc}0 & 1 \\ -1 & 0\end{array}\right)$

So, we obtain the spinor equations of Involute Evolute curves. After that, we will call the spinors corresponding to Involute Evolute curves as Involute Evolute spinors. The other relationship between Involute Evolute spinors can be given following theorem.

Theorem 3.4. Let the curves $\beta, \alpha: I \rightarrow \mathbb{E}^{3}$ be Involute Evolute curves and the spinors corresponding to the Frenet vectors of these curves are considered as $\phi$ and $\xi$, respectively. So, the relationship between Involute Evolute spinors is

$$
\xi^{t} \bar{\phi}^{t} \phi=\frac{1}{2}
$$

Proof. We know that for the spinors $\xi$ and $\phi$ there is relationship $\left|\xi_{1}\right|^{2}+\left|\xi_{2}\right|^{2}=1$ and $\left|\phi_{1}\right|^{2}+\left|\phi_{2}\right|^{2}=1$ since the Frenet vectors corresponding to these spinors are unit vectors. So, we can write

$$
\left(\left|\xi_{1}\right|^{2}+\left|\xi_{2}\right|^{2}\right)\left(\left|\phi_{1}\right|^{2}+\left|\phi_{2}\right|^{2}\right)=1
$$

So, we have

$$
\left|\xi_{1}\right|^{2}\left|\phi_{1}\right|^{2}+\left|\xi_{1}\right|^{2}\left|\phi_{2}\right|^{2}+\left|\xi_{2}\right|^{2}\left|\phi_{1}\right|^{2}+\left|\xi_{2}\right|^{2}\left|\phi_{2}\right|^{2}=1
$$

If we use the equations (3.8) and (3.9), we obtain

$$
\left(\xi_{1} \overline{\phi_{1}}+\xi_{2} \overline{\phi_{2}}\right)\left(\overline{\xi_{2}} \phi_{2}+\overline{\xi_{1}} \phi_{1}\right)=\frac{1}{2}
$$

Moreover, if the last equation is written by matrix product by the help of $\xi=\left(\begin{array}{c}\xi_{1} \\ \xi_{2}\end{array}\right), \phi=\left(\begin{array}{c}\phi_{1} \\ \phi_{2}\end{array}\right)$, the equation can be obtain

$$
\xi^{t} \bar{\phi}^{t} \phi=\frac{1}{2}
$$

Now, the expression of the Involute Evolute spinors in terms of each other can be written as follows.

Theorem 3.5. Let the spinors $\phi$ and $\xi$ be Involute Evolute spinors. So, the expression of spinor $\phi$ in terms of the spinor $\xi$ is

$$
\begin{aligned}
& \phi_{1}^{2}=\frac{\kappa-i \tau}{2 \sqrt{\kappa^{2}+\tau^{2}}}\left(\xi_{1}-\bar{\xi}_{2}\right)^{2} \\
& \phi_{2}^{2}=\frac{\kappa-i \tau}{2 \sqrt{\kappa^{2}+\tau^{2}}}\left(\bar{\xi}_{1}+\xi_{2}\right)^{2}
\end{aligned}
$$

Proof. We consider that the equations (3.4) and (3.5) are written in the second equation of (2.5). So, we find the equations

$$
\begin{aligned}
& \frac{1}{2}\left(\phi_{1}^{2}-\phi_{2}^{2}-\bar{\phi}_{2}^{2}+\bar{\phi}_{1}^{2}\right)=\frac{1}{\sqrt{\kappa^{2}+\tau^{2}}}\left(-\kappa\left(\xi_{1} \bar{\xi}_{2}+\bar{\xi}_{1} \xi_{2}\right)-i \frac{\tau}{2}\left(\xi_{1}^{2}-\xi_{2}^{2}+{\overline{\xi_{2}}}^{2}-\bar{\xi}_{1}^{2}\right)\right), \\
& \frac{1}{2}\left(\phi_{1}^{2}+\phi_{2}^{2}-\bar{\phi}_{1}^{2}-\bar{\phi}_{2}^{2}\right)=\frac{1}{\sqrt{\kappa^{2}+\tau^{2}}}\left(-\kappa\left(\xi_{1} \overline{\xi_{2}}-\bar{\xi}_{1} \xi_{2}\right)-i \frac{\tau}{2}\left(\xi_{1}^{2}+\xi_{2}^{2}+\bar{\xi}_{1}^{2}+\bar{\xi}_{2}^{2}\right)\right)
\end{aligned}
$$

and

$$
-\phi_{1} \phi_{2}-\bar{\phi}_{1} \bar{\phi}_{2}=\frac{1}{\sqrt{\kappa^{2}+\tau^{2}}}\left(-\kappa\left(\xi_{1} \bar{\xi}_{1}-\xi_{2} \bar{\xi}_{2}\right)+i \tau\left(\xi_{1} \xi_{2}-\overline{\xi_{1} \xi_{2}}\right)\right)
$$

Similarly, if the equations (3.4) and (3.5) are written in the third equation of (2.5), we find that

$$
-\frac{i}{2}\left(\phi_{1}^{2}-\phi_{2}^{2}+\bar{\phi}_{2}^{2}-\bar{\phi}_{1}^{2}\right)=\frac{1}{\sqrt{\kappa^{2}+\tau^{2}}}\left(\tau\left(\xi_{1} \overline{\xi_{2}}+\overline{\xi_{1}} \xi_{2}\right)-i \frac{\kappa}{2}\left(\xi_{1}^{2}-\xi_{2}^{2}+{\overline{\xi_{2}}}^{2}-{\overline{\xi_{1}}}^{2}\right)\right)
$$




$$
-\frac{i}{2}\left(\phi_{1}^{2}+\phi_{2}^{2}+\bar{\phi}_{1}^{2}+\bar{\phi}_{2}^{2}\right)=\frac{1}{\sqrt{\kappa^{2}+\tau^{2}}}\left(\tau\left(\xi_{1} \bar{\xi}_{2}-\bar{\xi}_{1} \xi_{2}\right)-i \frac{\kappa}{2}\left(\xi_{1}^{2}+\xi_{2}^{2}+{\overline{\xi_{1}}}^{2}+\bar{\xi}_{2}^{2}\right)\right)
$$

and

$$
i\left(\phi_{1} \phi_{2}-\bar{\phi}_{1} \bar{\phi}_{2}\right)=\frac{1}{\sqrt{\kappa^{2}+\tau^{2}}}\left(\tau\left(\xi_{1} \bar{\xi}_{1}-\xi_{2} \bar{\xi}_{2}\right)-i \kappa\left(-\xi_{1} \xi_{2}+\overline{\xi_{1} \xi_{2}}\right)\right) .
$$

If we consider the equations (3.10) and (3.13), we obtain

$$
\phi_{1}^{2}-\phi_{2}^{2}=\frac{\kappa-i \tau}{2 \sqrt{\kappa^{2}+\tau^{2}}}\left(\left(\xi_{1}-\overline{\xi_{2}}\right)^{2}-\left(\overline{\xi_{1}}+\xi_{2}\right)^{2}\right) .
$$

Similarly, from the equations (3.11) and (3.14), we have

$$
\phi_{1}^{2}+\phi_{2}^{2}=\frac{\kappa-i \tau}{2 \sqrt{\kappa^{2}+\tau^{2}}}\left(\left(\xi_{1}-\overline{\xi_{2}}\right)^{2}+\left(\overline{\xi_{1}}+\xi_{2}\right)^{2}\right) .
$$

Finally, from the equations (3.12) and (3.15), we get

$$
\phi_{1} \phi_{2}=\frac{\kappa-i \tau}{2 \sqrt{\kappa^{2}+\tau^{2}}}\left(\xi_{1}-\overline{\xi_{2}}\right)\left(\overline{\xi_{1}}+\xi_{2}\right) .
$$

So, we can write

$$
\begin{aligned}
& \phi_{1}^{2}=\frac{\kappa-i \tau}{2 \sqrt{\kappa^{2}+\tau^{2}}}\left(\xi_{1}-\bar{\xi}_{2}\right)^{2} \\
& \phi_{2}^{2}=\frac{\kappa-i \tau}{2 \sqrt{\kappa^{2}+\tau^{2}}}\left(\bar{\xi}_{1}+\xi_{2}\right)^{2}
\end{aligned}
$$

Now, we give an example.

Example 3.6. Let the unit-speed curve $\alpha: I \rightarrow \mathbb{E}^{3}$ be $\alpha(s)=\left(\frac{1}{\sqrt{2}} \cos s, \frac{1}{\sqrt{2}} \sin s, \frac{1}{\sqrt{2}} s\right)$. So, if we use the equation (2.2), for the Frenet vectors $\{\boldsymbol{T}, \boldsymbol{N}, \boldsymbol{B}\}$ of the curve $(\alpha)$, we calculate as

$$
\begin{aligned}
& \boldsymbol{T}(s)=\left(-\frac{1}{\sqrt{2}} \sin s, \frac{1}{\sqrt{2}} \cos s, \frac{1}{\sqrt{2}}\right), \\
& \boldsymbol{N}(s)=(-\cos s,-\sin s, \quad 0), \\
& \boldsymbol{B}(s)=\left(\frac{1}{\sqrt{2}} \sin s,-\frac{1}{\sqrt{2}} \cos s, \frac{1}{\sqrt{2}}\right) .
\end{aligned}
$$

Moreover, from the equation (2.3) we obtain the curvature and torsion of this curve as

$$
\kappa=\frac{1}{\sqrt{2}}, \quad \tau=\frac{1}{\sqrt{2}} .
$$

Now, we consider that the Frenet vectors $\{\boldsymbol{N}, \boldsymbol{B}, \boldsymbol{T}\}$ are corresponded to the spinor $\xi$. So, from the equations (3.4) and (3.16), we get

$$
\begin{aligned}
& \xi_{1}=\frac{1}{2} \sqrt{\frac{2+\sqrt{2}}{2}}(\sqrt{1-\cos s}+i \sqrt{1+\cos s}), \\
& \xi_{2}=-\frac{1}{2} \sqrt{\frac{2+\sqrt{2}}{2}}(\sqrt{1+\cos s}+i \sqrt{1-\cos s}),
\end{aligned}
$$

In addition that, from Theorem 2.6, we have $\frac{d \xi}{d s}=\frac{\sqrt{2}}{4}(-i \xi+\hat{\xi})$.

Now, we regard that the involute curve of unit-speed curve $(\alpha)$ is $(\beta)$ which has not arc-length parameter. So, if we look the equation (2.4), then the curve $(\beta)$ is written by

$$
\beta(s)=\frac{1}{\sqrt{2}}(\cos s-(c-s) \sin s, \sin s+(c-s) \cos s, c) .
$$

Then, we obtain the Frenet vectors and curvature, torsion of this curve

$$
\begin{aligned}
& \boldsymbol{T}^{*}(s)=(-\cos s,-\sin s, 0), \\
& \boldsymbol{N}^{*}(s)=(\sin s,-\cos s, 0), \\
& \boldsymbol{B}^{*}(s)=(0,0,1)
\end{aligned}
$$

and

$$
\kappa^{*}=\frac{\sqrt{2}}{(c-s)}, \quad \tau^{*}=0
$$

Finally, we get the spinor corresponded to involute curves

$$
\begin{aligned}
& \phi_{1}=\frac{1}{2}(\sqrt{1+\sin s}+i \sqrt{1-\sin s}), \\
& \phi_{2}=-\frac{1}{2}(\sqrt{1-\sin s}+i \sqrt{1+\sin s}) .
\end{aligned}
$$

So, the Frenet equation of this curve in terms of a single spinor equation is written by $\frac{d \phi}{d s}=\frac{1}{2} \hat{\phi}$. 


\section{References}

[1] É. Cartan, The Theory of Spinors, The M.I.T. Press, Cambridge, MA, 1966.

[2] H. B. Lawson, M. L. Michelsohn, Spin Geometry, Princeton University Press, New Jersey, 1989.

[3] P. O’Donnell, Introduction to 2-Spinors in General Relativity, World Scientific Publishing Co. Pte. Ltd., London, 2003.

[4] M. D. Vivarelli, Development of spinors descriptions of rotational mechanics from Euler's rigid body displacement theorem, Celestial Mech., 32 (1984), $193-207$.

[5] G. F. T. Del Castillo, G. S. Barrales, Spinor formulation of the differential geometry of curves, Rev. Colombiana Mat., 38 (2004), $27-34$.

[6] I. Kişi, M. Tosun, Spinor Darboux equations of curves in Euclidean 3-space, Math. Morav., 19(1) (2015), 87-93.

[7] D. Unal, I. Kisi, M. Tosun, Spinor Bishop equation of curves in Euclidean 3-space, Adv. Appl. Clifford Algebr., 23(3) (2013), $757-765$.

[8] Z. Ketenci, T. Erisir, M.A. Gungor, A construction of hyperbolic spinors according to Frenet frame in Minkowski space, J. Dyn. Syst. Geom., Theor 13(2) (2015), 179-193.

[9] T. Erisir, M. A. Gungor, M. Tosun. Geometry of the hyperbolic spinors corresponding to alternative frame, Adv. Appl. Clifford Algebr., 25(4) (2015), $799-810$.

[10] Y. Balci, T. Erisir, M. A. Gungor, Hyperbolic spinor Darboux equations of spacelike curves in Minkowski 3-space, J. Chungcheong Math. Soc., 28(4) (2015), 525-535.

[11] M. Tarakcioglu, T. Erisir, M. A. Gungor, M. Tosun. The hyperbolic spinor representation of transformations in $\mathbb{R}_{1}^{3}$ by means of split quaternions, Adv. Appl. Clifford Algebr., 28(1) (2018), 26.

[12] H. H. Hacisalihoglu, Differential Geometry, Faculty of Science, Ankara University, 1, 1996.

[13] H. Goldstein, Classical Mechanics, 2nd ed., Addison-Wesley, Reading, Mass., 1980.

[14] D. H. Sattinger, O. L. Weaver, Lie Groups and Algebras with Applications to Physics, Geometry and Mechanics, Springer-Verlag, New York, 1986.

[15] W. T. Payne, Elementary spinor theory, Amer. J. Phys., 20 1952, 253. 